\title{
Patient satisfaction following benign forehead mass excision through a direct or remote approach
}

\section{Won Hyuck Do, Young Woong Choi}

Department of Plastic and Reconstructive Surgery, Inje University Sanggye Paik Hospital, Inje University College of Medicine, Seoul, Korea
Background Benign tumors of the forehead are highly prevalent and can cause facial asymmetry, discomfort, and psychological issues for patients. Generally, excision is performed via an incision directly over the mass, which can produce suboptimal cosmetic outcomes. Many methods involving remote incisions have been developed to conceal scars at the hairline. This study compared patient satisfaction after remote and direct incisional approaches.

Methods We retrospectively enrolled 122 patients who underwent forehead mass excision at our clinic between January 2010 and May 2019 and compared the remote and direct removal of benign forehead lesions. Data on demographics, tumor size, operative time, imaging method, the incidence of complications, and pathological results were collected. Patient satisfaction was assessed via a telephone survey. The complications monitored included hypoesthesia due to nerve injury, wound dehiscence or necrosis, hematoma or seroma, and recurrence.

Results A total of 79 patients underwent direct-approach mass excision, and 43 underwent excision with a remote approach. Lipoma was the most common tumor (70 patients), followed by osteoma (26 patients). Statistical analyses with the Mann-Whitney and Fisher exact tests revealed significantly higher satisfaction among the remote-approach group than among the direct-approach group $(\mathrm{P}<0.05)$.

Conclusions From the perspective of scaring and complication, the group that removed forehead mass through remote approach had significantly better results than the group that removed through direct approach. However, patients' perception of scar visibility strongly influenced their satisfaction. Therefore, a remote approach should be considered for patients who are concerned about cosmetic outcomes, and the patient's hairstyle should also be considered.

Keywords Forehead / Lipoma / Osteoma

\section{INTRODUCTION}

The forehead is the most prominent facial region due to its convexity. Therefore, asymmetry and scarring of the forehead cause pa-

Received: Jul 15, 2020 Revised: Aug 6, 2020 Accepted: Aug 9, 2020 Correspondence: Young Woong Choi Department of Plastic and Reconstructive Surgery, Inje University Sanggye Paik Hospital, Inje University College of Medicine, 1342 Dongil-ro, Nowon-gu, Seoul 01757, Korea Tel: +82-2-950-1048, Fax: +82-2-932-6373, E-mail: pshero@gmail.com Copyright @ 2020 The Korean Society for Aesthetic Plastic Surgery. This is an Open Access article distributed under the terms of the Creative Commons At tribution Non-Commercial License (https://creativecommons.org/licenses/by-nc/4.0/) which permits unrestricted non-commercial use, distribution, and reproduction in any medium, provided the original work is properly cited. $\quad w w w . e-a a p s . o r g$ tients more concern than cosmetic issues in other regions. Depressed facial scars can occur on any part of the face, but their presence on the forehead often results in psychological and aesthetic effects [1].

The forehead is a common site of benign tumors, such as osteoma and lipoma [2]. The most common site of cranial osteomas is the frontal sinus; however, osteomas that involve only the frontal bone periosteum are occasionally observed [3]. Lipomas also frequently occur in the forehead region; superficial subcutaneous lipomas are likely underreported, and they have been depicted on the faces of kings minted onto coins as far back as 32-37 BC [4].

The increased interest over recent years in these forehead tumors and excisional scars has led to the development of various surgical methods for their removal. The gold standard for removing tumors of the forehead is direct incision over the mass. However, the di- 
rect-incision approach results in conspicuous scars; therefore, alternative approaches represent an important area of research. Remote (indirect) approaches for head and neck surgery involving instruments such as endoscopes were first described by Gagner [5]. The remote approach using trocars is preferred by patients despite the challenging nature of the surgical procedure, presumably because the scars are adjacent to the hairline and are not visible. In the present study, we investigated the satisfaction of patients whose scars were covered by a hairstyle involving "bangs" following a direct surgical approach. Many studies have compared the remote approach to the direct approach, but few studies have investigated patient satisfaction. Furthermore, this study is the first to consider differences in patient satisfaction according to hairstyle, and we believe that our results will be of great help to craniofacial surgeons.

\section{METHODS}

The study was approved by the Institutional Review Board of Inje University Sanggye Paik Hospital (IRB No. 2020-04-029) and performed in accordance with the principles of the Declaration of Helsinki. We retrospectively reviewed the records of patients who underwent excision of benign masses on the forehead in the plastic surgery department of our institution between January 2010 and May 2019. The exclusion criterion was epidermoid cysts that contained a central punctum upon inspection; these cysts can only be removed using direct excision because the incision must include the central core or punctum [6]. The follow-up time points included the day after surgery, twice per week until the removal of stitches, and 6 months after the removal of stitches. Patients with masses diagnosed as osteomas, lipomas, pilomatrixomas, granulomas, hemangiomas, and other benign tumors were included in this study. Patients were categorized into either the direct-approach or the remote-approach group according to the procedure used. From a retrospective review of electronic medical records, we collected patient data including demographic information, preoperative and postoperative photographs, surgical records, surgical approach, anesthetic procedures, operative time, length of the tumor, whether drainage was required, pathologic outcomes, complications, admission day, and last outpatient visit.

Patient satisfaction surveys were conducted via telephone at least 6 months postoperatively. The survey involved a questionnaire composed of the following four questions: (1) Did similar lesions occur adjacent to the original tumor? (2) Did the patient experience complications, such as hypoesthesia, after surgery, and did the complications improve? (3) Is the scar covered by the hairstyle at present (6 months after surgery)? (4) What is the patient's satisfaction score regarding the procedure? Patient satisfaction was scored from 0 to 10 points based on the patient's subjective satisfaction. When investigating the patient satisfaction score, we assessed whether the scar was covered by the patient's hair, regardless of the sur- gical approach, and whether the scar could be disguised using a hairstyle with bangs or a long and straight hairstyle. The patient satisfaction scores were analyzed according to the hairstyle and scars at the time of the telephone survey. We also divided the remote-approach group into a group that underwent an endoscopic response and a group that underwent non-endoscopic surgery. In the endoscopic approach, a single incision of a length equivalent to the diameter of the mass was applied to the hairline closest to the mass, and a single-port approach was used. A total of 28 patients experienced mass excision that involved an endoscope, and 15 patients underwent mass excision with no endoscope. The patient satisfaction score was also compared between these two subgroups.

Radiological examinations, such as computed tomography or ultrasonography, were not required in the vast majority of cases; these modalities were used only for tumors that were large, deep, or vague even after sufficient preoperative physical examination. Preoperative computed tomography was carried out to evaluate the differentiation of encapsulated versus non-encapsulated lipomas [7] and to rule out the intracranial extension of dermoid cysts [8].

The patient's hairstyle was assessed through clinical pictures of the patient and the telephone survey. Intraoperative photographs and data were retrospectively reviewed based on electronic medical records.

The surgical approach was selected based on the size, depth, and characteristics of the tumor as well as the distance from the hairline, the patient's preference, and the patient's hairstyle. No hair of any patient was cut or shaved. The remote approach was performed under general endotracheal or local anesthesia. After surgical marking directly above the mass, a solution of $1 \%$ lidocaine with 1:200,000 epinephrine was infiltrated into the galea or periosteum for hydrodissection. One horizontal incision was made just below the hairline at a location that provided easy access to the tumor at the shortest distance possible. The dissecting plane was determined based on the location and characteristics of the tumor. In cases involving osteomas or lipomas attached to the frontal bone, the incision was made down to the subperiosteal plane. Other tumors were primarily dissected in the subgaleal plane. If the mass was above the galea, the galea was dissected, and the tumor was bluntly mobilized. Generally, a sheet retractor was used to visualize the tumor in the remote approach; endoscopic equipment was only used in cases where the tumor was not accessible due to a limited visual field or the shortness of the sheet retractor. After dissection and resection of the tumor, profuse irrigation was carried out to remove debris or remnant hair using a saline solution. In the case of osteomas, all osteomas existed only on the outer cortex without invading the inner cortical bone and were removed through the osteotome to enable biopsy. The frontal bone was smoothened using a curved rasp. All incisions were closed with simple interrupted sutures that were removed after 10 to 14 days (Fig. 1).

The direct approach involved making a surgical mark on the 

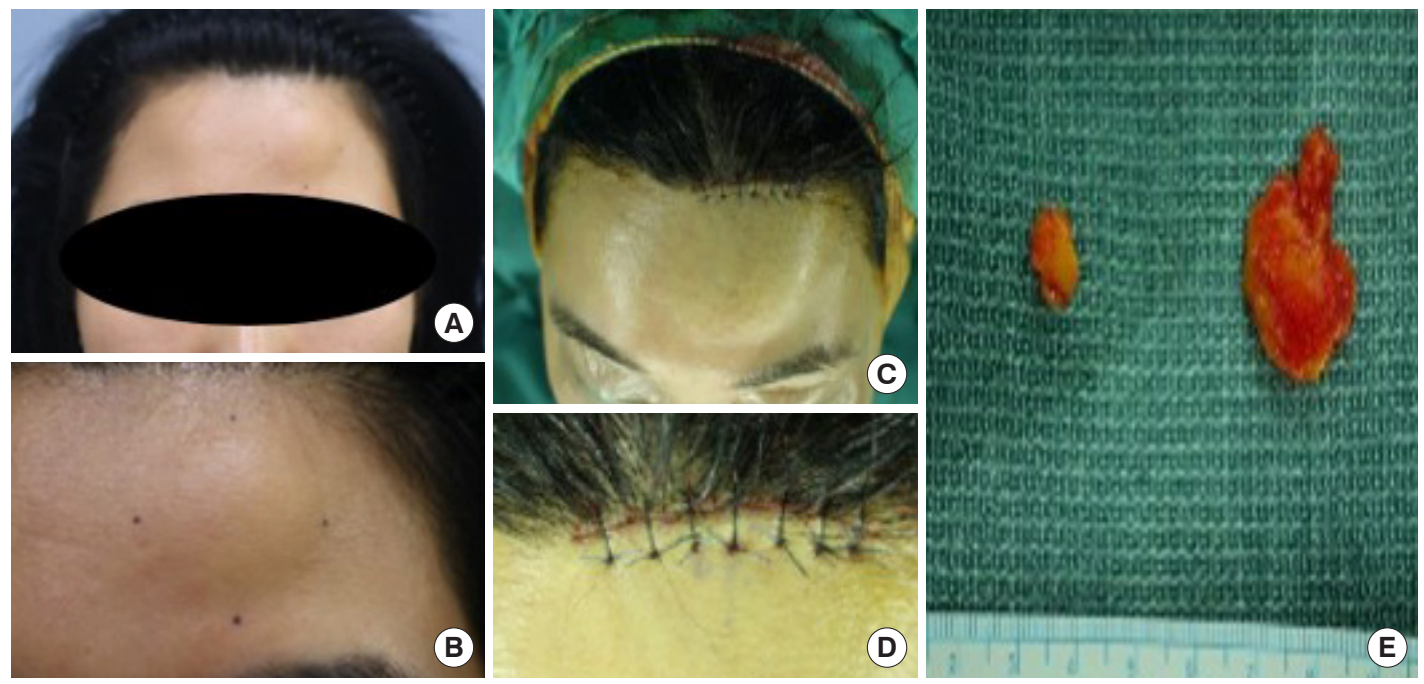

Fig. 1. Preoperative $(A, B)$ and postoperative $(C, D)$ photographs of tumor excision via a remote approach. The incision was made at the patient's hairline. After reaching the superior border of the mass, the periosteum was sharply incised, and further blunt dissection of the tumor was performed in the supraperiosteal or subgaleal plane. An endoscope was used if the mass was far from the hairline. (E) Specimen of the forehead mass.
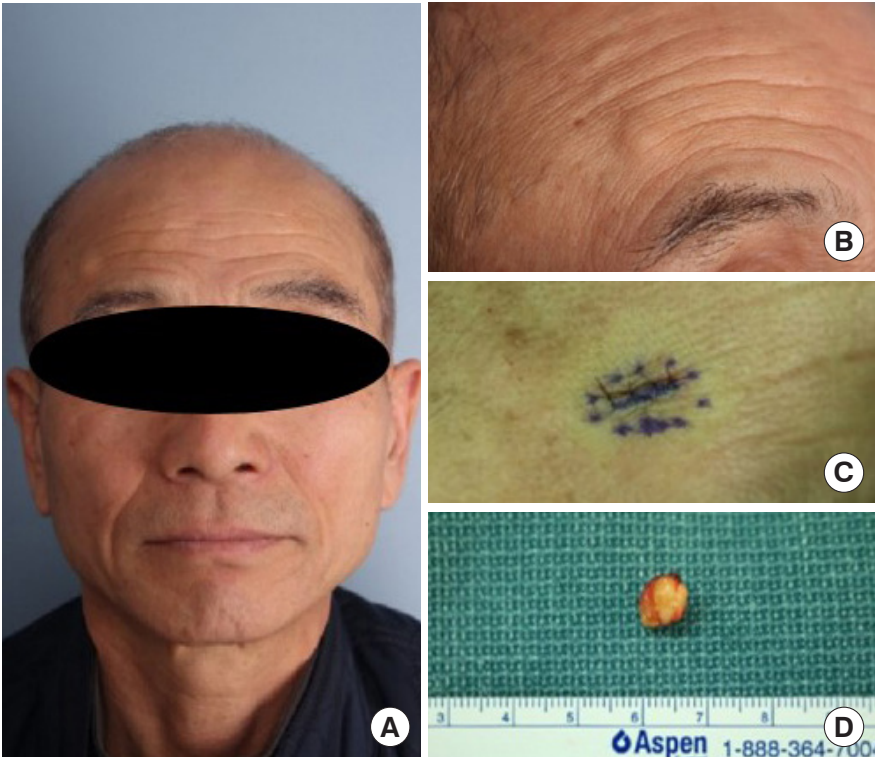

A
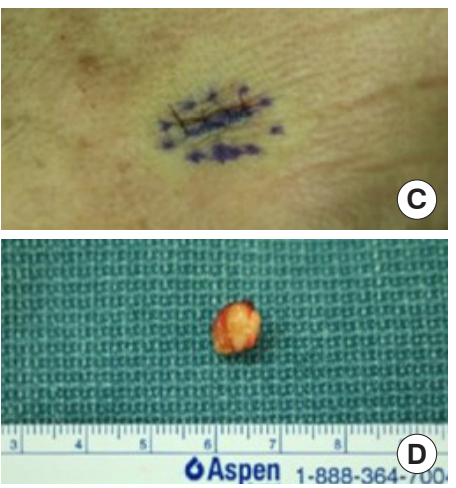

Fig. 2. Preoperative $(A, B)$ and postoperative $(C)$ photographs of direct tumor excision via an open approach. An overlying horizontal skin incision has been described as the classic resection method, and this method inevitably leaves a visible scar even in patients with an asymptomatic clinical course. (D) Lipoma specimen of the forehead.

skin followed by an incision along the relaxed skin tension line directly above the mass. The length of the incision was designed to be approximately two-thirds of the length of the mass. After resection, incisions were closed with sutures that were removed after 5 to 10 days (Fig. 2). All excised tumors were sent to the pathology department for histological examination. Taping was performed for 1 week after the removal of stitches. In cases where the remote approach was used, a silicone sheet could not be applied due to the presence of hair, and a silicone gel ointment was mainly used. In contrast, in the cases involving a direct approach, gel-type and sheettype silicone treatment were simultaneously administered. In each group, scar management was performed for 6 months in the same fashion. No patients underwent laser therapy or scar revision.

Among pediatric patients, surgery was performed under local anesthesia if the patient was cooperative and tolerated local injection. However, if the child was too young or unable to cooperate, general anesthesia was administered, and scar evaluation was performed by the guardian.

Categorical data are presented as numbers (\%), and continuous data are presented as means. The Fisher exact test and the MannWhitney test were used to compare the mean data of the categorical variables between the direct-approach and remote-approach groups. A P-value of $<0.05$ was considered to indicate statistical significance. Statistical analyses were performed using SPSS version 22 (IBM Corp., Armonk, NY, USA).

\section{RESULTS}

We recruited a total of 210 patients with benign forehead tumors, but 88 patients could not be contacted or refused to answer the survey and were excluded. A final total of 122 patients were retrospectively enrolled in our study. The direct-approach group included 79 patients, and the remote-approach group included 43 patients. Table 1 details the demographic information for the study population according to group. The P-values are the values obtained by comparing the direct-approach group with the remote-approach 
Table 1. Demographic characteristics of the direct-approach and remote-approach groups

\begin{tabular}{|c|c|c|c|c|}
\hline Variable & $\begin{array}{c}\text { Direct } \\
\text { approach } \\
\text { [n=79] }\end{array}$ & $\begin{array}{l}\text { Remote } \\
\text { approach } \\
\text { (n=43) }\end{array}$ & $\begin{array}{c}\text { Total study } \\
\text { population } \\
\text { (n=122) }\end{array}$ & P-value \\
\hline Sex & & & & 0.002 \\
\hline Male & $44(55.6)$ & 11 (25.5) & $55(45.1)$ & \\
\hline Female & 35 (44.3) & $32(74.4)$ & $67(54.9)$ & \\
\hline Age, yr & $42.3 \pm 17.7$ & $45.6 \pm 12.1$ & 43.4 & 0.016 \\
\hline \multicolumn{5}{|l|}{ Pathological results } \\
\hline Lipoma & $48(60.7)$ & 21 (48.8) & $69(56.5)$ & \\
\hline Osteoma & $7(8.8)$ & $19(44.1)$ & 26 (21.3) & \\
\hline Hemangioma & $3(3.7)$ & $2(4.6)$ & $5(4.0)$ & \\
\hline Granuloma & $4(5.0)$ & 0 & $4(3.2)$ & \\
\hline Pilomatrixoma & $3(3.7)$ & 0 & $3(2.4)$ & \\
\hline Other tumors & $14(17.7)$ & $1(2.3)$ & 15 (12.2) & \\
\hline \multicolumn{5}{|l|}{ Anesthetic method } \\
\hline Local anesthesia & $72(91.1)$ & $22(51.1)$ & $94(77.0)$ & \\
\hline General anesthesia & $7(8.8)$ & 21 (48.8) & $28(22.9)$ & \\
\hline \multicolumn{5}{|l|}{ Radiologic evaluation } \\
\hline None & $24(30.3)$ & $6(13.9)$ & $30(24.5)$ & \\
\hline Computed tomography & $29(36.7)$ & 35 (81.3) & $64(52.4)$ & \\
\hline Ultrasonography & $26(32.9)$ & $2(4.6)$ & $28(22.9)$ & \\
\hline Follow-up period, yr & $5.49 \pm 2.67$ & $4.79 \pm 3.29$ & 5.25 & 0.259 \\
\hline \multicolumn{5}{|l|}{ Tumor length, $\mathrm{cm}$} \\
\hline Long axis & $1.38 \pm 0.76$ & $1.51 \pm 0.74$ & 1.43 & 0.268 \\
\hline Short axis & $1.06 \pm 0.70$ & $1.17 \pm 0.67$ & 1.10 & 0.263 \\
\hline Operation time, $\min$ & $29.00 \pm 16.47$ & $34.70 \pm 48.46$ & 31.00 & 0.099 \\
\hline Satisfaction score & $7.28 \pm 1.64$ & $8.95 \pm 1.13$ & 7.86 & $<0.001$ \\
\hline Complications & 3 & 2 & 5 & 0.580 \\
\hline Recurrence & 1 (33.3) & $1(50.0)$ & $2(40.0)$ & \\
\hline Hypoesthesia & 1 (33.3) & $1(50.0)$ & $2(40.0)$ & \\
\hline Wound problem & 1 (33.3) & 0 & $1(20.0)$ & \\
\hline
\end{tabular}

Values are presented as number $(\%)$ or mean \pm SD.

group.

All histological examinations were based on results diagnosed by a pathologist through histopathologic assessment. Lipomas were the most common tumor in both groups. In the direct-approach group, local anesthesia was used for the vast majority of patients; general anesthesia was used in very young patients or upon specific request by the patient. In contrast, the use of general anesthesia was more frequent in the remote-approach group than in the directapproach group; the type of anesthesia was selected based on tumor size and depth. The mean short and long axes of the tumor, as well as the operative time, were slightly greater in the remote-approach group than in the direct-approach group, although the difference in these parameters was not statistically significant between

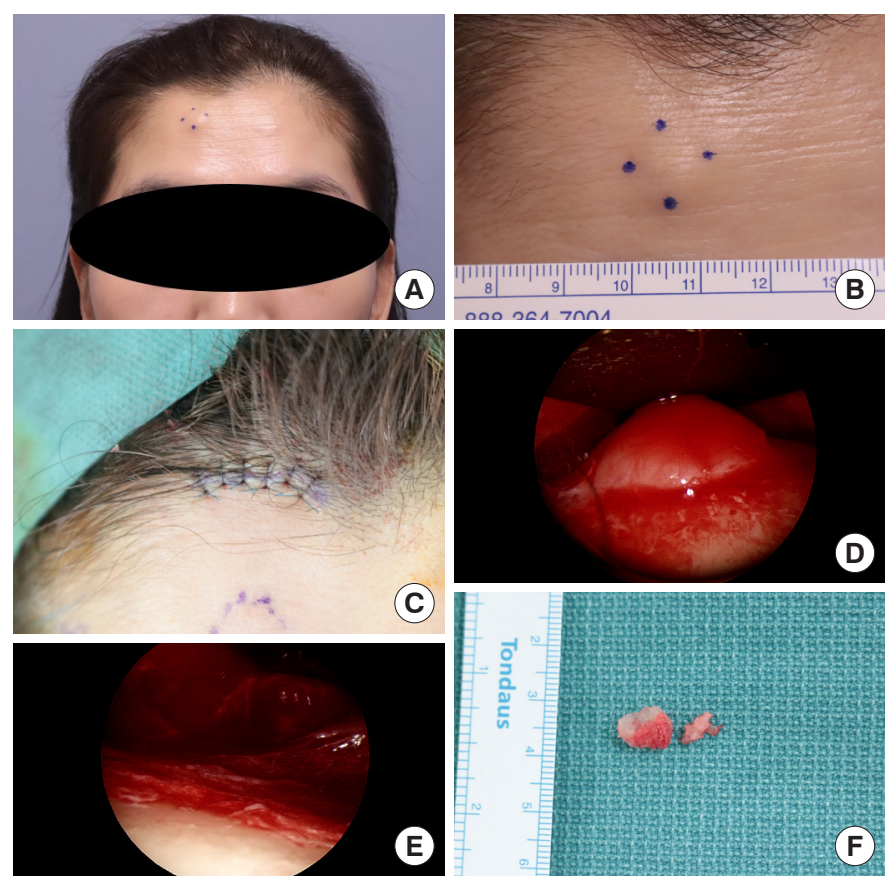

Fig. 3. Preoperative $(A, B)$ and postoperative (C) photographs of hairline incision via an endoscopic approach. (D) Endoscopic photograph of osteoma. (E) Endoscopic photograph of the frontal bone after resection of osteoma. (F) Osteoma specimen.

the groups.

The mean satisfaction score of the remote-approach group was significantly higher than that of the direct-approach group. Subdivision of the remote-approach group according to the utilization of an endoscope revealed that an endoscope was used for 28 of the 43 patients (Fig. 3). The mean satisfaction score of the endoscopic subgroup $(9.00 \pm 1.15)$ was higher than that of the non-endoscopic subgroup (8.87 \pm 1.12$)$, although the difference was not statistically significant $(\mathrm{P}=0.642)$.

A total of 31 patients had scars that were hidden by their hairstyles and were referred to as "covered." A total of 91 patients had a hairstyle that did not cover their scars and were referred to as "uncovered." The mean satisfaction score of the covered patients was $8.65 \pm 1.17$, which was significantly higher than that of the uncovered patients $(7.60 \pm 1.75)(\mathrm{P}=0.008)$.

The admission decision after surgery was made by comprehensively considering the size of the mass, the presence of drainage, and the patient's needs. In the direct-approach group, 10 patients were hospitalized, and the average hospital stay was 2.5 days. In the remote-approach group, 20 patients were hospitalized, and the average hospital stay was 3.2 days. The P-value was 0.159 , which did not indicate a statistically significant difference. Since the criteria for hospitalization and discharge were different for each surgeon, it is not surprising that these results were not statistically significant.

Postoperative complications were more common in the direct- 
approach group than in the remote-approach group. One case of wound margin necrosis due to postoperative hematoma was reported in the direct-approach group, and this was resolved by evacuation through stitch removal, squeezing, and the application of a compressive dressing. One lipoma recurred as a small tumor, but the patient refused excision. One patient suffered hypoesthesia at the area of supraorbital nerve innervation, but the hypoesthesia started to improve after a few weeks, and the patient completely recovered sensation. In the remote-approach group, one patient suffered localized numbness at the area of supratrochlear nerve innervation immediately after surgery; this resolved completely after 1 year. One lipoma recurred, but the patient refused excision of the recurrent tumor due to its small size. No cases of motor deficits were observed in either group. The incidence of complications was not significantly different between the groups.

\section{DISCUSSION}

The upper third of the face is of unique importance for the overall facial aesthetics. Studies of eye movement have demonstrated that, when observers are asked to gauge the age and fatigue of study subjects, the area of the face that is most scrutinized by observers is the periorbital region [9]. Therefore, the role of the forehead in determining the overall impression of a person cannot be overlooked. In addition, a scar that is clearly visible on the forehead is likely to have psychological effects on the individual. Therefore, several methods have been developed to conceal scars in this area.

The gold-standard treatment for benign forehead masses has been excision using a direct approach. However, this approach leaves noticeable scars, which can include skin pigmentation and hypertrophic scars; therefore, remote approaches have been devised. The utility of liposuction and narrow-hole extrusion techniques has been reported, but these techniques are not commonly used due to their disadvantages, which include poor visualization and high recurrence rates [10]. In recent years, mass removal using a remote approach has become more commonly used, as the concealable incision leads to improved aesthetic outcomes. In addition, the use of an endoscope enables good visualization and a magnified view, which can help the surgeon protect important anatomical structures [11].

As can be seen from the results of the present study, statistically significant differences were present with regard to sex and age. It can be assumed that female patients tend to prefer the remote approach because they are more sensitive to scars than male patients. In the direct-approach group, children including a 1-year-old and a 9-year-old were included, but in the remote-approach group, the minimum age was 24 years old, and we presume that this led to the statistically significant difference that was observed. We found that the satisfaction rate of the patients who underwent remote surgery was significantly higher than that of the patients who underwent surgery via a direct approach. This is most likely due to the fact that the scar from the remote approach is located on the hairline. Therefore, the scar is covered by the patient's hair, and the mass is generally considered to have been removed scarlessly; this results in less severe psychological effects, as the scar will be invisible to others. Interestingly, among the patients who reported that their scars were not visible, not all scars were completely covered by their hair. We found that, regardless of the approach used, patient satisfaction was high when the patient believed the scar was concealed by their hair, even when the scar was not completely covered. This implies that even partial concealment of the scar leads patients to believe that it is less noticeable to others, which results in improved psychological stability and increased confidence.

The remote approach is usually performed in the subgaleal or subperiosteal plane, which depends on the location and depth of the mass and the surgeon's preference. Each plane has advantages; for example, the subgaleal plane offers a slightly more superficial approach and a wider operative field, and the procedure can be performed quickly and easily with reduced blood loss due to the ability to more directly access the soft tissue [12]. Therefore, the protocol at our center is to remove all masses except for those directly attached to the frontal bone via a subgaleal-plane approach.

In the case of a remote approach, the possibility of general anesthesia or monitored anesthesia care is increased. Due to the limited visualized field, hard bleeding control would prolong the operation time. Also, when using a remote approach without an endoscope, the incision line usually becomes much longer than a direct incision. In addition, in the case of a remote approach, the range of dissection increases to reach the lesion. In this case, drainage may be required, and the hospitalization period may be lengthened. Also, swelling can be more severe than when using a direct approach.

The drawback of the remote approach is that the surgical time is longer. However, in our study, we did not find a statistically significant difference between the remote and direct approaches; this may be due to the skill of our experienced surgeons and the reduced time required for suturing due to the shorter incision required for the remote approach. In addition, the direct approach is performed through a small incision to minimize the scar, which results in poor visualization of the mass and more time required to complete the procedure [13]. Furthermore, even when using a remote approach, we found no cases in which bleeding was a problem after meticulous hemostasis and compressive dressing. In addition, management through silastic drainage was easy after discharge, so no significant difference was present in the short hospitalization period $(\mathrm{P}=0.159)$.

In this study, we identified no significant difference in the frequency of complications between the groups and found that most complications could be prevented through sufficient irrigation, careful hemostasis, and the use of a compressive dressing of folded gauze and an elastic head bandage. In addition, our study involved no 
cases of suspected neurovascular bundle injury following remote surgery. Care must be taken to remain in the appropriate plane during dissection because the supraorbital and supratrochlear neurovascular nerves follow the superficial layer of the frontalis muscle. Other drawbacks of the remote approach are that it requires expensive endoscopic equipment and involves a demanding surgical technique.

The present study has some limitations that should be acknowledged. First, selection bias may have been present because patients were not randomized to the type of surgery that they received. The smoothness of the patients' foreheads, surgeon's preference, patients' cosmetic desires, and cost of the operations were comprehensively considered preoperatively. A prospective randomized study is warranted and could provide a more credible comparison of the different surgical approaches.

Finally, the hairline of an individual changes with age. However, Cronin et al. [14] pointed out that the remote approach is also applicable for men with a sparse or receding hairline; however, the authors emphasize that future hairline changes may necessitate a more posteriorly-placed incision [1].

In conclusion, when removing a forehead mass, the remote approach is considered to be a superior surgical treatment method because the patient satisfaction is high and no significant difference exists with regard to the incidence of complications. Determining the surgical method by considering the patient's hairstyle and awareness of scarring will greatly help to increase patient satisfaction after surgery. In addition, our results will help plastic surgeons determine the surgical method according to the characteristics of the forehead mass.

\section{NOTES}

\section{Conflict of interest}

YWC, the editor-in-chief of the Archives of Aesthetic Plastic Surgery, is the corresponding author of this article. However, he played no role whatsoever in the editorial evaluation of this article or the decision to publish it. Except for that, no potential conflict of interest relevant to this article was reported.

\section{Ethical approval}

The study was approved by the Institutional Review Board of Inje University Sanggye Paik Hospital (IRB No. 2020-04-029) and performed in accordance with the principles of the Declaration of Helsinki.

\section{Patient consent}

The patients provided written informed consent for the publication and the use of their image.

\section{ORCID}

Won Hyuck Do

Young Woong Choi

https://orcid.org/0000-0003-1114-2488

https://orcid.org/0000-0002-2211-2981

\section{REFERENCES}

1. Ozinko MO, Otei OO, Ekpo RG, et al. Depressed forehead scar: a case report and review of literature. J Surg Case Rep 2019;2019:rjz368.

2. Sadick H, Huber M, Perkins SW, et al. Endoscopic forehead approach for minimally invasive benign tumor excisions. JAMA Facial Plast Surg 2014;16:352-8.

3. Patel TR, Borah GL. Frontal bone periosteal osteomas. Plast Reconstr Surg 2004;114:648-51.

4. Grosshans EM. Subfascial lipoma of the forehead. J Am Acad Dermatol 1990;23:153-4.

5. Gagner M. Endoscopic subtotal parathyroidectomy in patients with primary hyperparathyroidism. Br J Surg 1996;83:875.

6. Hoang VT, Trinh CT, Nguyen CH, et al. Overview of epidermoid cyst. Eur J Radiol Open 2019;6:291-301.

7. Do WH, Choi YW. Complications of the surgical excision of encapsulated versus nonencapsulated lipomas: a retrospective analysis. Arch Aesthetic Plast Surg 2019;25:142-6.

8. Dutta S, Lorenz HP, Albanese CT. Endoscopic excision of benign forehead masses: a novel approach for pediatric general surgeons. J Pediatr Surg 2006;41:1874-8.

9. Garritano FG, Quatela VC. Surgical anatomy of the upper face and forehead. Facial Plast Surg 2018;34:109-13.

10. Christenson L, Patterson J, Davis D. Surgical pearl: use of the cutaneous punch for the removal of lipomas. J Am Acad Dermatol 2000;42: 675-6.

11. Im JT, Park BY, Lim SY, et al. Endoscopic excision of benign facial tumors: a decade of experience with 152 patients. Ann Plast Surg 2014; 72:556-9.

12. Lopez M, Vermersch S, Varlet F. Endoscopic excision of forehead and eyebrow benign tumors in children. J Laparoendosc Adv Surg Tech A 2016;26:226-30.

13. Jung S, Jung SW, Koh SH, et al. Forehead mass removal by endoscopic approach. J Craniofac Surg 2016;27:e215-7.

14. Cronin ED, Ruiz-Razura A, Livingston CK, et al. Endoscopic approach for the resection of forehead masses. Plast Reconstr Surg 2000;105:245963. 